\title{
DETECCIÓN DE CAMBIOS NO SUPERVISADA DE IMÁGENES SAR PARA EL SEGUIMIENTO DEL COMPORTAMIENTO Y ANÁLISIS DE INUNDACIONES EN COLOMBIA
}

\author{
DETECTION OF UNSUPERVISED SAR IMAGES CHANGES \\ FOR THE MONITORING OF THE BEHAVIOR \\ AND ANALYSIS OF FLOODS IN COLOMBIA
}

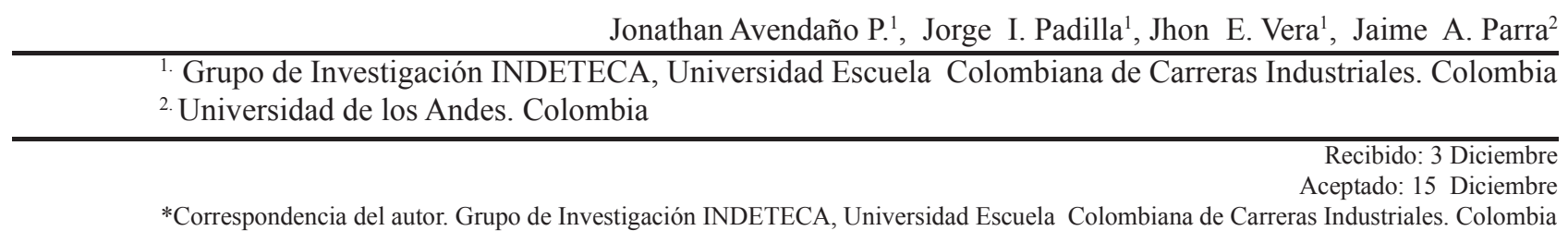

\section{RESUMEN}

Este trabajo propone un método no supervisado de detección cambios para el estudio del comportamiento de las inundaciones en Colombia usando imágenes SAR (Synthetic Aperture Radar). El enfoque de detección de cambios propuesto es un método pre-Clasificación, en el cual se clasifican primero las imágenes y luego por medio de operadores de relación se encuentra los cambios. En primera instancia se filtraron las imágenes SAR con el filtro Frost-Mejorado con el objetivo de eliminar el efecto del ruido Speckle. Para la segmentación no supervisada de imágenes SAR se utilizaron los enfoques de Fuzzy-Clustering (FCM) y k-means, los resultados de ambos enfoques fueron fusionados con el fin de mejorar la precisión en la segmentación usando el método de fusión de imágenes PCA (Principal Component Analysis). Para la detección de cambios se implementaron los operadores de relación Mean-Ratio y Log-Ratio, los resultados de ambos operadores de relación también fueron fusionados usando PCA con el fin de mejorar la precisión en esta fase del método propuesto. Para la experimentación se usaron en primera instancia imágenes sintéticas creadas a partir de zonas homogéneas de imágenes SAR de Colombia. Con estas imágenes fue posible evaluar el desempeño del método propuesto. Los resultados mostraron una precisión total alrededor del $99 \%$, lo que concluye que el método propuesto es altamente eficiente sobre el conjunto de datos usado. Luego se experimentó sobre dos imágenes SAR reales, una antes y otra después de una inundación, las cuales fueron proporcionadas por el IDEAM y cubren el área del Magdalena bajo en el municipio del Plato-Magdalena. El método propuesto encontró que en estas imágenes aproximadamente 77.46 hectáreas del municipio del Plato- Magdalena fueron inundadas por el río Magdalena.

Palabras claves: SAR, Segmentación, Detección de cambios, imágenes de zonas de inundación. 


\begin{abstract}
This paper proposes an unsupervised method of detecting changes for studying the behavior of the floods in Colombia using images SAR (Synthetic Aperture Radar). The proposed change detection approach is a pre-classification method, in which first images are classified and then changes are found through relational operators. First, SAR images were filtered with filter-Enhanced Frost in order to eliminate the effect of Speckle noise. For unsupervised segmentation of SAR images, the Fuzzy Clustering (FCM) approach and k-means were used. The results from both approaches were fused in order to improve accuracy in segmentation using the method of image fusion PCA (principal Component Analysis). For the detection of changes, operators ratio Mean-Log-Ratio Ratio were implemented, the results of both relational operators were also fused using PCA in order to improve accuracy in this phase of the proposed method. For the experiment, first, synthetic images created from homogeneous areas of SAR images of Colombia were used. With these images, it was possible to evaluate the performance of the proposed method. The results showed an overall accuracy about 99\%, which concludes that the proposed method is highly efficient on the data set used. Then, two real SAR images were experimented, one before and one after a flood, which were provided by the IDEAM and cover the area of Magdalena in the Plato-Magdalena. The proposed method found in these images about 77.46 hectares in the municipality of Magdalena plate were flooded by the Magdalena River..
\end{abstract}

Keywords: SAR Segmentation, detection of changes, images floodplain.

\section{Introducción}

En Colombia debido a su ubicación geográfica las inundaciones son muy comunes. Estas inundaciones traen consigo pérdidas humanas y económicas que afectan directamente los intereses del país. Casi todo el territorio nacional en épocas de lluvia se ve afectado por este fenómeno, pero sin duda alguna las zonas más afectadas son aquellas que se encuentran cercanas a los ríos, lagunas, embalses, ciénagas etc. La zona conocida como el magdalena bajo es la más afectada en épocas de lluvia. En varios años las inundaciones en esta parte del territorio Colombiano han costado la vida de muchas personas y han afectado la agricultura y la ganadería, principales fuentes económicas en este territorio. Las inundaciones son un proceso el cual es muy rápido en su fase de inundación pero muy lento a la hora de retirarse el agua del territorio inundado.

Durante varios años se ha evidenciado el uso de los datos multi-temporales para el seguimiento de las inundaciones y diferentes fenómenos naturales, tal es el caso del trabajo realizado en 2010 por Xu et al. (1) donde se mostró la efectividad de fusionar imágenes multi-temporales SAR polarimétricas con imágenes

Rev. Invest. Univ. Quindío. (Col.), 27(2): 54-61; 2015 ópticas de alta resolución como las que provee el sensor QuickBird, en su trabajo los autores demostraron que la fusión de estos datos mejora notablemente la clasificación de coberturas de tierra y el efecto del ruido Speckle sobre las imágenes SAR. Por otro lado en agosto de 2011, Pul- virenti et al. (2), realizaron un trabajo que busca estudiar el fenómeno de las inundaciones por medio del uso de datos multi-temporales Cosmo SkyMed. En china, en el año de 2012 Ban \& Yousif, (3), realizaron un trabajo de investigación cuyo objetivo era el de examinar la efectividad de los métodos de detección de cambios en zonas urbanas y observar la rápida expansión de china. En 2013, Paul and Ramamoorthy (4), realizaron un algoritmo para la detección de cambios de imágenes SAR usando FCM y los operadores de relación Mean-Ratio y LogRatio. Es evidente el uso de la detección de cambios en imágenes SAR para el seguimiento de fenómenos naturales como por ejemplo las inundaciones. Como se puede observar la tendencia es usar métodos preclassification como el que se expone en este trabajo.

El Global Climate Risk Index es un reporte en el cual se exponen los índices de riesgo climático global, este informe es publicado anualmente por la Organización No Gubernamental (ONG) alemana Germanwatch y es de acceso libre. En este reporte se muestra por 
medio de cifras el impacto causado por los eventos relacionados con el cambio climático (inundaciones, tormentas, olas de calor, etc.) sobre algunos países. Esta organización cuenta con datos estadísticos y demográficos que datan desde 1991 a la fecha. En el último informe que corresponde al año 2014 (5), en el ranking histórico desde el año 1991 hasta el 2012 Colombia ocupa el puesto número 42 a nivel mundial en países afectados por el cambio climático, la principal causa son las inundaciones. En el ranking del 2012 el cual se muestra en este informe Colombia ocupa el puesto 25 a nivel mundial; sin embargo, la situación más crítica se produjo en el año 2010 cuando Colombia ocupo el puesto número 3 a nivel mundial, este año corresponde a la ruptura del canal del dique. Como se observa las inundaciones son una problemática que afecta directamente a Colombia por esta razón en la actualidad se busca con las nuevas tecnologías en sistemas de información geográfica monitorear diferentes zonas en riesgo de inundaciones. En Colombia la ola invernal provoca frecuentemente muchos desastres. Diferentes organizaciones del estado Colombiano poseen información espacial importante que con un debido procesamiento puede entregar una herramienta para la evaluación, prevención y seguimiento de las inundaciones. En 2010 la comisión nacional del espacio colombiano realizó un estudio a nivel nacional con las empresas públicas y privadas sobre la necesidad de la adquisición de imágenes satelitales y fortalecimiento de los procesos de investigación y desarrollo de tecnologías de observación de la tierra por medio de sensores remotos (6). Motivados en este estudio y en sus objetivos que evidencian la necesidad de investigar y desarrollar tecnologías que usen imágenes satelitales y sumado a la problemática que causan las inundaciones en Colombia se realiza este trabajo que usa imágenes satelitales tipo SAR, llamadas así debido a que son obtenidas por medio de un radar de apertura sintética. Estas imágenes juegan un papel importante a la hora de evaluar cualquier fenómeno natural sobre la tierra. Su mayor ventaja sobre otro tipo de imágenes es que pueden ser tomadas en cualquier condición climática y de tiempo. Este trabajo propone un método no supervisado de detección cambios para el estudio del comportamiento de las inundaciones en Colombia usando imágenes SAR, este método se divide en varias fases: filtrado (filtro Frost); detección de diferencias usando los algoritmos de Mean-Ratio y Log-Ratio; fusión temporal de imágenes usando el enfoque PCA; segmentación no supervisada usando los algoritmos de k-means y FCM; binarización o umbralización y ecualización del histograma. El método propuesto busca integrar las ventajas de dos diferentes métodos en detección de cambios, Mean-Ratio y Log-Ratio. Se propone implementar por ventanas cada uno de estos algoritmos con la finalidad de obtener mejor precisión en la detección de cambios. Este enfoque es un método pre-classification en el cual es importante el proceso de segmentación, por esta razón en este trabajo también se fusiona los resultados de dos de las técnicas de segmentación no supervisada comúnmente usadas, FCM y k-means con el objetivo de mejorar la precisión en la detección de zonas inundadas.

\section{Método propuesto}

El método que se presenta en este trabajo fue implementado en MATLAB. Para la experimentación en primera instancia se generaron dos imágenes de referencia creadas a partir de zonas homogéneas de imágenes SAR reales, estas imágenes sirvieron para evaluar el desempeño del método propuesto. En segunda instancia se utilizaron imágenes tipo SAR del territorio Colombiano para el estudio del comportamiento del río, Magdalena, debido a que estas son independientes al estado climático o de tiempo en el que son tomadas, lo que permite tener una buena observación de la superficie terrestre sin la afectación de las nubes como en el caso de las imágenes ópticas. En la figura 1 se ilustra el método propuesto para la fusión temporal y detección de cambios.

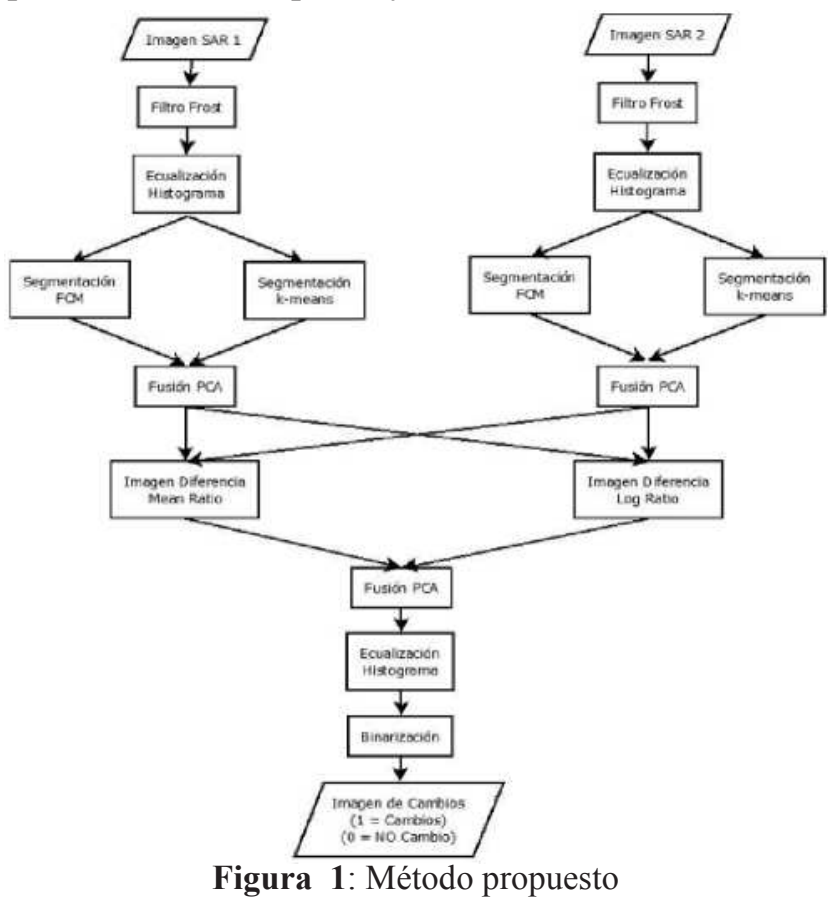

Figura 1: Método propuesto 


\subsection{Etapa de Filtrado}

En esta etapa se procesan las imágenes para reducir el ruido Speckle. El filtro Frost es un algoritmo adaptativo propuesto por Frost et al. (7), en 1982, basado en el error cuadrático medio que se adapta a las estadísticas de la imagen, este algoritmo asume que el ruido es multiplicativo y aleatorio. Basa su funcionamiento en el filtro adaptativo de Wiener (8), el cual asume que el ruido y la imagen son un proceso aleatorio y su objetivo es buscar un estimativo ( error cuadrático medio entre dos imágenes.

\subsection{Etapa de Detección de Diferencias (Cambios)}

En esta etapa por medio de los operadores MeanRatio y Log-Ratio se encuentra las diferencias entre la imagen 1 y la imagen 2 . Al final de esta etapa se tendrán dos imágenes diferencia resultado de aplicar los dos operadores en ambas imágenes. Debido a que el ruido Speckle es aleatorio sobre toda los píxeles de la imagen, para la implementación de ambos enfoques de detección de cambios se utilizaran ventanas de 9x9. Lo anterior permite evaluar cada píxel con base a sus vecinos para determinar si este píxel cambia de una imagen a otra o simplemente es un píxel ruidoso proveniente del ruido Speckle. Se podría entonces decir que la detección de cambios propuesta en este método se realiza por medio de regiones. De cada ventana se calcula el promedio y con este se evalúa por medio de los enfoques de Mean-Ratio y Log-Ratio si existe o no cambios en esa región de la imagen.

\subsubsection{Operador Mean-Ratio}

El funcionamiento de este enfoque consiste en hallar la relación entre las medias de dos imágenes multitemporales. La razón de trabajar con las medias es con el fin de reducir el efecto del ruido Speckle sobre las imágenes SAR. La fórmula que describe este operador de relación, por ventanas, es la siguiente:

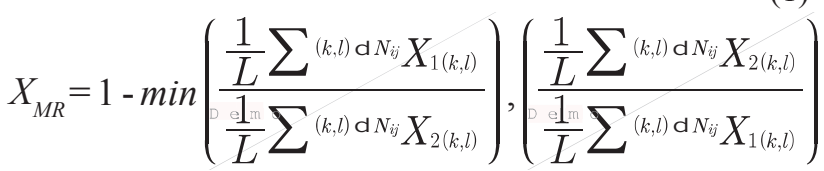

Donde Nij denota los píxeles vecinos del píxel (i, j) dentro de la ventana y (L) el tamaño de la ventana. Al escoger el mínimo entre las relaciones de las medias de las ventanas se garantiza que los valores resultantes estén en un rango de 0 a 1 . Con base en la ecuación (1) este enfoque entrega un 0 cuando no existe un cambio entre las ventanas de las imágenes evaluadas, y valores mayores que 0 y menores que 1 cuando existe un cambio, identificando las zonas donde más existen cambios cuando el resultado es cercano a 1.

\subsubsection{Operador Log-Ratio}

Este operador de relación a diferencia del operador Mean-Ratio el cual basa su funcionamiento en calcular la relación que existe entre los valores de las medias locales de dos imágenes multi-temporales SAR, se enfoca en calcular la relación logarítmica existente entre dos imágenes multi-temporales. Con el operador Log-Ratio, el ruido multiplicativo Speckle puede ser transformado en un componente de ruido aditivo. El operador Log-Ratio el cual busca encontrar las diferencias entre dos imágenes multitemporales, se define por la siguiente ecuación:

$X L R=\log \left(\frac{\frac{1}{L} \sum(k, l) \mathrm{d} N_{i j}}{\frac{1}{L} \sum_{2(k, l)}(k, l) \mathrm{d} N_{i j} X_{1(k, l)}}\right)$

La escala logarítmica se caracteriza por el aumento del valor de los píxeles con baja intensidad y la disminución del valor de los píxeles de alta intensidad, por lo tanto, la distribución de dos clases (cambio y no cambio) se podría hacer más simétrica. Sin embargo, la información de las regiones modificadas que se obtiene por el operador Log-Ratio puede no ser capaz de reflejar la verdadera tendencia de cambio en la mayor medida posible a causa de la disminución del valor de los píxeles en las áreas con alta intensidad dentro de la imagen.

\subsection{Fusión Temporal de Imágenes}

La fusión de imágenes se usa para combinar información procedente de diferentes fuentes con el fin de entregar una imagen de mayor calidad que las imágenes de entrada cuando estas se consideran de forma aislada. Pajares \& Cruz (9). En este trabajo la fusión se utiliza en dos partes: la primera para fusionar las imágenes diferencia resultado de los operadores Mean-Ratio y Log-Ratio; y la segunda para fusionar los resultados de las segmentaciones de los métodos de FCM y k-means. La razón de realizar un proceso de fusión es la de complementar la información 
en cada fase del método propuesto en este trabajo; por ejemplo obtener una imagen diferencia que una las ventajas de cada operador y una imagen segmentada con mayor precisión. Como la fusión se realiza sobre imágenes con la misma resolución espacial pero con diferente resolución temporal se considera como Fusión Temporal.

El enfoque fusión escogido para implementar en este trabajo fue el basado en análisis por componentes principales (PCA). Este método consiste realizar una transformación orto normal de dos imágenes de entrada cuya dimensión es de $(N \times M)$. El algoritmo de PCA implementado es explicado en (9).

\subsection{Ecualización del Histograma}

La ecualización del histograma es una técnica que consiste en ajustar el nivel de la escala de grises de una imagen. Esta técnica se basa en una transformación del histograma con el fin de proporcionar una imagen cuyo histograma sea uniforme y bien distribuido. Esta etapa se realiza en dos partes del método propuesto: la primera después de la etapa de filtrado con el objetivo de evitar falsas diferencias en la etapa de detección de cambios, estas falsas diferencias pueden producirse si las imágenes procesadas no tienen una distribución de niveles de grises similar debido a que por ejemplo provienen de diferentes sensores remotos; la segunda vez que se aplica esta etapa es después de la fusión de los resultados de las dos técnicas de segmentación, que se mencionan más adelante, con el fin de separar bien las clases (firmas espectrales) que entrega este proceso.

\subsection{Fase de Segmentación}

La segmentación busca extraer características de las imágenes como: puntos de interés, bordes, contornos, regiones, etc. Este proceso divide la imagen en grupos de píxeles u objetos dando como resultado un conjunto de segmentos que cubren la imagen, o un conjunto de curvas de nivel extraídas de la imagen. Dass et al. (10). FCM y k-means son métodos de segmentación basados en Clustering los cuales buscan agrupar píxeles en Clusters. Los algoritmos de FCM y $k$-means son enfoques interactivos que de manera autónoma agrupan datos dentro de las imágenes entregando como resultado una imagen segmentada en colores, textura, intensidad o una mezcla de los anteriores. Avendaño \& Parra (11). Los algoritmos de segmentación FCM y k-means implementados en este proyecto son explicados en (11).

\subsection{Fase de Binarización}

Esta es la última etapa del método propuesto y busca etiquetar cada píxel de la imagen resultante con 1 cuando existe cambio y 0 cuando no. Para esto se usa la segmentación por umbral conocida como bancarización debido a que la imagen resultante solo comprende valores Booleanos (0 y 1$)$. (9)

\section{Experimentación y Resultados}

En este capítulo se experimentara sobre el método propuesto. La experimentación tendrá dos partes: la primera consistir en evaluar cada fase del proceso y evaluar su desempeño usando dos imágenes de referencia; la segunda parte consistirá en emplear el método propuesto sobre imágenes SAR de Colombia.

\subsection{Imágenes de Referencia}

Para poder evaluar el desempeño del método propuesto y de cada una de sus fases fue necesario crear dos imágenes sintéticas a partir de zonas homogéneas de las imágenes SAR de Colombia utilizadas en este estudio. Estas imágenes al ser creadas por el usuario permiten conocer los cambios exactos entre ellas, permitiendo a lo último evaluar los resultados que entrega el método propuesto.

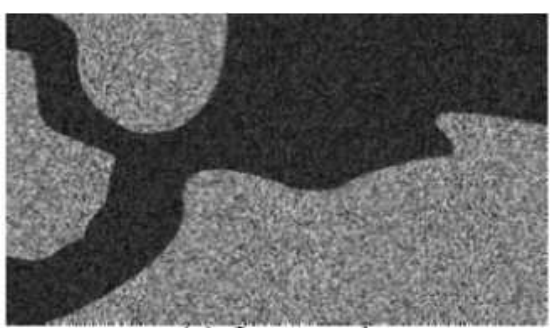

(a) Imagen I

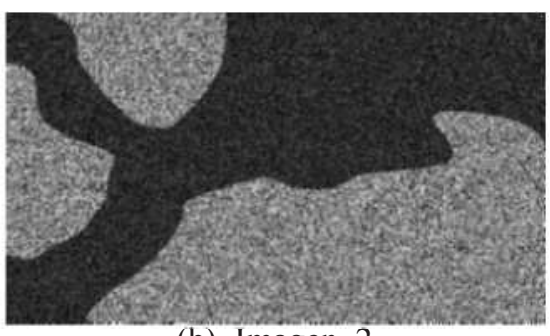

(b) Imagen 2

Figura 2: Imágenes de Referencia 
Estas imágenes tienen un tamaño de 460x819 píxeles y se crearon con base en una imagen real tratando de simular un río y los terrenos cercanos al mismo. Las imágenes de las figura (2) solo tienen dos grupos de píxeles, los correspondientes a tierra y los correspondientes a agua. Cabe resaltar que estas imágenes de referencias al ser creadas de imágenes SAR traen consigo ruido Speckle.

\section{2. Índices de evaluación}

Con el fin de evaluar el desempeño de la metodología propuesta se hará uso de la matriz de confusión y de los índices Overall Accuracy y Kappa, (12). Estos índices miden la eficiencia del método a la hora de encontrar diferencias. El efecto del ruido Speckle sobre las imágenes hace que se generen falsos positivos en la detección, este método busca en primera instancia eliminar este efecto sobre las imágenes, pero se sabe que esto no es del todo suficiente, por eso en el método de detección diferencias implementado se implementaron ventanas con la finalidad de tener en cuenta la información de los píxeles vecinos de la imagen.

\subsection{Detección de cambios de las imágenes de referencia}

A continuación se muestran los resultados encontrados de aplicar el método propuesto aplicando 3 veces el filtro Frost a las imágenes de referencia y utilizando 2 cluster para la segmentación.

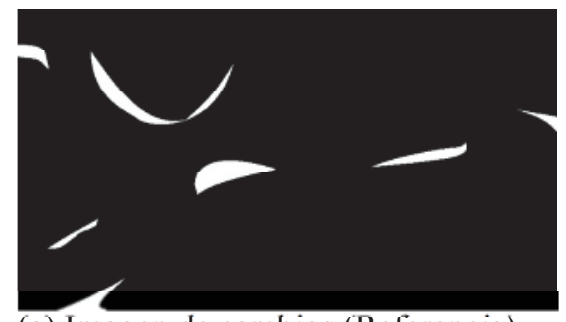

(a) Imagen de cambios (Referencia)

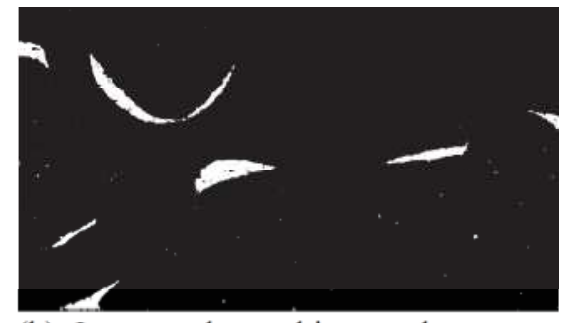

(b) Imagen de cambios resultante

Figura 3: Imagen de cambios de referencia vs. Imagen encontrada
Los píxeles blancos indican cambios en la imagen, mientras que los negros indican lo contrario. Al evaluar el resultado método propuesto se obtuvo un Overall Accuracy del 99.4 \% y un índice Kappa de 0.89 que indican un nivel de precisión alto y un índice de concordancia casi perfecto. Los valores anteriores fueron tomados de la matriz de confusión que se genera al comparar la imagen de cambios (referencia) y la imagen de cambios resultante. Estos resultados también pueden ser comprobados de forma visual en la figura (3), donde se evidencia que la imagen de cambios que entrega el método propuesto es muy parecida a la de la referencia.

\subsection{Caso de Estudio}

En las secciones anteriores se evaluó la precisión del método propuesto usando imágenes de referencia, ahora es momento de implementar el método propuesto sobre imágenes reales de zonas Colombianas. La zona escogida fue la del magdalena bajo en el municipio del Plato Magdalena. Se escogió esta zona del territorio colombiano debido a que esta zona es fuertemente afectada por el tema de las inundaciones. Una de las imágenes utilizadas corresponde a una inundación que se presentó en el año de 2010, época en la que se rompió el canal del dique y que trajo bastantes estragos para Colombia. La otra imagen utilizada corresponde a esta misma zona pero en épocas donde no está inundado. Las imágenes utilizadas provienen del sensor remoto RADARSAT-2, tiene polarización HH, una resolución radiométrica de 16 bits y una resolución espacial de 10 metros. Estas imágenes fueron suministradas por el IDEAM para el desarrollo de este proyecto. Las imágenes entregadas por el IDEAM cubren gran parte del río magdalena razón por la cual tuvieron que ser cortadas para enfocarse en el caso de estudio.

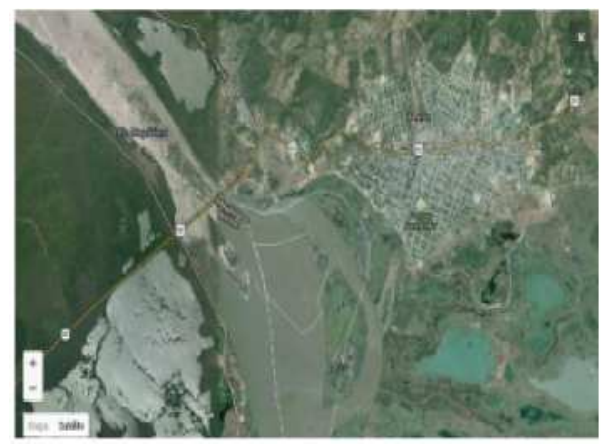

Figura 4: Plato Magdalena - Fuente: Google Earth 
En la figura 4 se ilustra una imagen satelital Landsat del municipio del plato magdalena, esta imagen fue tomada de Google Earth. Como se puede observar este municipio está realmente cercano a las orillas del río Magdalena lo que lo hace vulnerable cuando el nivel de este río aumenta. La ubicación del este municipio se encuentra en 79॰ 77' de longitud occidental y 9॰ y 48' de latitud norte. El clima promedio en esta zona de Colombia es de $32 \circ \mathrm{C}$ y la altura promedio sobre el nivel del mar es de 20 metros. Para detectar los cambios del río se hará uso del método propuesto. A continuación se ilustran las imágenes usadas y los resultados obtenidos.

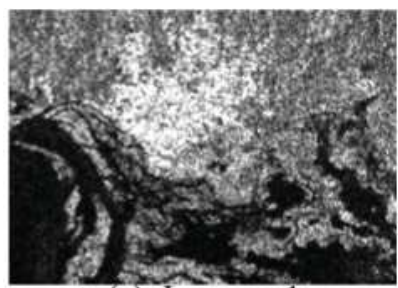

(a) Imagen 1

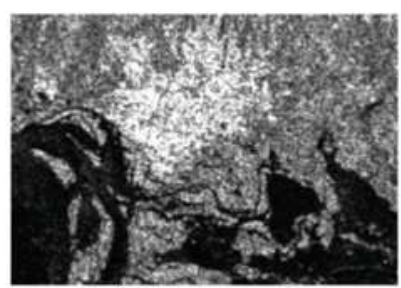

(b) Imagen 2

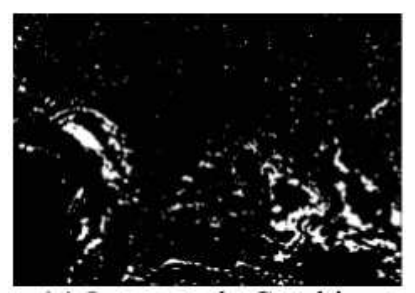

(c) Imagen de Cambios

Figura 5: Imágenes de caso de estudio

En la figura 5 se muestran los resultados de aplicar el método propuesto sobre las imágenes del caso de estudio. Las imágenes (1) y (2) tienen un tamaño de 312x4446 píxeles, como se conoce la resolución espacial de estas imágenes es posible determinar el área de cambios dentro de la imagen; como la resolución espacial es de 10 metros esto quiere decir que cada píxel dentro de la imagen representa $100 \mathrm{~m} 2$. El resultado encontrado fue que el área de cambios que encontró el método propuesto es de $1,315,100 \mathrm{~m} 2$, lo que equivale a que 131,51 Hectáreas de 138,715 cambiaron entre las dos imágenes

\section{Conclusión}

Este trabajo presenta un método para la detección de cambios pre-classification en zonas de inundación en Colombia utilizando imágenes SAR. En primera instancia se filtraron las imágenes con el objetivo de eliminar el efecto del ruido Speckle característico en dichas imágenes, después se realizó un proceso de segmentación para agrupar los píxeles que corresponden a la clase agua y finalmente se detectó los cambios entre las imágenes usando operadores de relación, Mean-Ratio y Log-Ratio. Los resultados obtenidos al aplicar el método propuesto sobre imágenes de referencia, creadas a partir de zonas homogéneas de imágenes SAR de Colombia, arrojaron una precisión del $99.4 \% \mathrm{y}$ un índice de concordancia de 0.89 lo que concluye que el método propuesto es altamente eficiente para la detección de cambios sobre el conjunto de datos usado. Al aplicar el método propuesto sobre las imágenes, una antes y otra después, del caso de estudio correspondientes a una inundación que se presentó en el año de 2010 en la zona del Magdalena bajo se encontró que 77.46 hectáreas del municipio del Plato-Magdalena fueron inundadas por el río.

\section{Agradecimientos}

Agradecemos al IDEAM por las imágenes SAR que facilitaron para el desarrollo de este trabajo. 


\section{BIBLIOGRAFÍA}

1. Xu M, Xia Z, Zhang F, Li K, Xie C. Multi-Temporal Polarimetric SAR and Optical Data Fusion for Land Cover Mapping in Southwest China. In: Multimedia Technology (ICMT), 2010 International Conference on; 2010. p. 1-4.

2. Pulvirenti L, Chini M, Pierdicca N, Guerriero L, Ferrazzoli P. Flood monitoring using multi- temporal COSMO-SkyMed data: Image segmentation and signature interpretation. Remote Sen- sing of Environment. 2011;115(4):990 - 1002. Available from: http://www.sciencedirect.com/ science/article/ pii/S0034425710003408.

3. Ban Y, Yousif OA. Multitemporal Spaceborne SAR Data for Urban Change Detection in China. Selected Topics in Applied Earth Observations and Remote Sensing, IEEE Journal of. 2012 Aug;5(4):1087-1094.

4. Paul L, Ramamoorthy DP. Synthetic Aperture Radar Image Change Detection Using Fuzzy CMeans Clustering Algorithm. International Journal of Advanced Research in Computer and Communication Engineering. 2013 March;2:1374 - 1379.

5. Kreft S, Eckstein D. Global Climate Risk Index 2014. Germanwatch; 2013.

6. Arias LP, Santacruz AM, Posada E. Programa satelital colombiano de observación de la Tierra: una estrategia de innovación y desarrollo tecnológico para Colombia. Análisis Geográficos. 2010;(44):176.

7. Frost VS, Stiles JA, Shanmugan KS, Holtzman J. A Model for Radar Images and Its Application to Adaptive Digital Filtering of Multiplicative Noise. Pattern Analysis and Machine Intelligence, IEEE Transactions on. 1982 March;PAMI-4(2):157-166.

8. Wiener N. Extrapolation, Interpolation, and Smoothing of Stationary Time Series. The MIT Press; 1964.

9. Pajares G, de la Cruz Garcia JM. Ejercicios resuletos de Vision por Computador. Mexico D.F., 221 Mexico: Alfaomega; 2008.

10. Dass R, Priyanka, Devi S. Image Segmentation Techniques. International Journal of Electronics \& Communication Technology IJEC. 2012 March;3(14):66-70.

11. Avendaño J, Parra J. Segmentación y clasificación de imágenes SAR en zonas de inundación en Colombia, una herramienta computacional para prevención de desastres. INGE@UAN. 2014;4(8).

12. Liu C, Frazier P, Kumar L. Comparative assessment of the measures of thematic classification accuracy. Remote Sensing of Environment. 2007;p. 606-616. 